\title{
Urothelial cancer of the prostate gland. Immunohistochemical analysis of two cases of rare canine cancer
}

\author{
Rafal Ciaputa $^{1}$, Valeria Grieco ${ }^{2}$, Izabela Janus ${ }^{1}$, Malgorzata Kandefer-Gola ${ }^{1}$, \\ Piotr Dziegiel $^{3,4}$, Marcin Nowak ${ }^{1}$
}

${ }^{1}$ Department of Pathology, Division of Pathomorphology and Veterinary Forensics, Faculty of Veterinary Medicine, Wroclaw University of Environmental and Life Sciences, Wroclaw, Poland

${ }^{2}$ Department of Veterinary Medicine, Università degli Studi di Milano, Milan, Italy

${ }^{3}$ Division of Histology and Embryology, Department of Human Morphology and Embryology, Wroclaw Medical University, Wroclaw, Poland

${ }^{4}$ Department of Physiotherapy, Wroclaw University School of Physical Education, Wroclaw, Poland

\begin{abstract}
Introduction. The study aimed to diagnose urothelial cancer of prostate gland (UCPG) in dogs as the primary focus and in its metastasis based on the expression of specific proteins used in immunohistochemical diagnosis of prostate cancer in men.

Material and methods. The study was carried out on specimens collected during a post-mortem examination from macroscopic lesions of the prostate glands from two dogs. The immunoexpression of the following proteins was verified: prostate-specific antigen (PSA), high molecular weight cytokeratins (HMWCK), cytokeratin 7 and 20 (CK-7,-20), E-cadherin, von Willebrand factor, cyclooxygenase-2 (COX-2), microsomal PGE2-1 synthase (mPGES-1) and component of the minichromosome 7 maintenance complex (MCM7).

Results. All markers, except for PSA, were expressed both at the primary tumour site and in the metastasis. Conclusions. The immunohistochemical approach was more useful for the diagnosis of UCPG in dog than typical histopathological staining methods because it allowed for precise determination of features, type and grade of the tumour that may affect its early detection and treatment. (Folia Histochemica et Cytobiologica 2020, Vol. 58, No. 4, 272-277)
\end{abstract}

Key words: canine; prostate; urothelial cancer; metastasis; cell markers; IHC

\section{Introduction}

A tendency towards an increasing number of hyperplastic prostate lesions and tumours was reported both in humans and animals. Among the neoplastic prostate lesions, prostatic carcinoma is the most

\footnotetext{
Correspondence address: Rafal Ciaputa, DVM, PhD

Department of Pathology, Division of Pathomorphology and Veterinary Forensics,

Faculty of Veterinary Medicine,

Wroclaw University of Environmental and Life Sciences,

C.K. Norwida 31, 50-375 Wroclaw, Poland,

e-mail: rafal.ciaputa@upwr.edu.pl
}

life-threatening one. For example, its death rate in humans is 40000 per year in the USA [1]. Prostate cancer is diagnosed in $40 \%$ of men during routine autopsy [1]. Therefore, numerous papers suggested that canine prostate cancer is the best model to study the factors that affect initiation and progression of the prostate cancer in humans and animals that live in the same environment [2]. Prostatic carcinoma is most often diagnosed in dogs older than 10 years, irrespective of their breed or age [3].

After converting the age of dogs into that of humans, the time of incidence of prostate cancer in men and dogs seems similar (70 and 67 years, respectively) [3]. This tumour most often occurs in intact male dogs 
or early-neutered dogs, which may be associated with its hormone dependence [3]. Prostatic carcinoma in dogs and men is not only similar in terms of the age of occurrence but also terms of the metastatic loci. It is most often located in the skeletal system (lumbar and pelvic region), the surrounding lymph nodes, lungs, liver, and colon [3-5]. According to the World Health Organization (WHO), prostatic carcinoma in animals is divided into adenocarcinoma and poorly differentiated prostatic cancer [5, 6]. Prostatic urothelial carcinomas, derived from urethral epithelium or periurethral prostatic ducts, are rarely diagnosed $[5,6]$.

The study aimed to develop a method of early diagnosis of prostate cancer in dogs based on the expression of specific proteins used in immunohistochemical (IHC) diagnostics of prostate cancer in men. These included prostate-specific antigen (PSA), high molecular weight cytokeratins (HMWCK), cytokeratin 7 and 20 (CK-7, CK-20), as well as proteins allowing for tumour type determination, i.e. E-cadherin, von Willebrand factor, cyclooxygenase-2 (COX-2), microsomal PGE2 synthase-1 (mPGES-1) and minichromosome maintenance complex component 7 (MCM7). The examined panel of antibodies should allow for a thorough analysis of the origin of the tumour. It will allow to differentiate it into prostate cancer and urothelial cancer of prostate gland, as well as to determine the aggressiveness of the primary tumour and its metastases.

\section{Materials and methods}

The study was carried out on specimens collected during a post-mortem examination from macroscopic lesions of the prostate glands from two dogs. Metastatic lesions were found in the bladder of one of the dogs. The dog with bladder metastases was a German Shepherd, 10 years old, weighing $35 \mathrm{~kg}$, not castrated. The second case was a 12-year-old mixed-breed dog weighing $10 \mathrm{~kg}$, also uncastrated.

The collected specimens were fixed in $7 \%$ buffered formalin for $24 \mathrm{~h}$, then embedded in paraffin blocks and cut into $4 \mu \mathrm{m}$ sections. The sections were stained with haematoxylin and eosin and underwent histopathological assessment. The prostate tumours were graded using the WHO Animals classification of prostate cancer in dogs [6].

IHC stainings were carried out on $4 \mu \mathrm{m}$ thick paraffin sections placed on silanized slides $\left(\mathrm{DAKO}^{\circledR}\right.$ catalogue no. S 3003; Dako, Glostrup, Denmark). The sections were then deparaffinised using xylene and passed to water through decreasing alcohol concentrations. Tissue antigens fixed in formalin were unmasked using EnVision ${ }^{\mathrm{TM}}$ FLEX Target Retrieval Solution, high pH $\left(50 \times\right.$; DAKO ${ }^{\circledR}$ catalogue no. $\mathrm{K} 8004$ ) by heating in a water bath at $96^{\circ} \mathrm{C}$ for $20 \mathrm{~min}$. In the case of PSA, restriction digested with proteinase $\mathrm{K}$ was performed. Endogenous peroxidase was blocked with EnVision $^{\mathrm{TM}}$ FLEX Peroxidase-Blocking Reagent for 10 minutes. Next, primary antibodies were applied to the sections at following dilutions as listed in Table 1 . All the sections were incubated with primary antibodies at room temperature (RT) for $20 \mathrm{~min}$. Then, they were washed with EnVision ${ }^{\mathrm{TM}}$ FLEX Wash Buffer $(20 \times)$. Next, EnVision ${ }^{\text {TM }}$ FLEX/HR SM802 detection system was applied, and the sections were again incubated at RT for $20 \mathrm{~min}$. The IHC reaction was elicited using EnVision ${ }^{\mathrm{TM}}$ FLEX DAB + Chromogen DAKO 3,3-diaminobenzidine tetrahydrochloride (DAB) solution. The sections were then washed in distilled water. The cell nuclei were counterstained with haematoxylin and dehydrated in a series of increasing alcohol concentrations. The specimens were exposed to xylene and fixed in a mounting medium.

Positive and negative controls were performed for each cell marker. Positive controls were selected for individual markers from the samples obtained from a pathology laboratory, and their positivity was confirmed by comparing them with other samples. For this purpose, specimens from mammary gland cancer, prostate cancer, normal skin tissue and tonsils were used. In the negative controls primary antibodies were omitted.

Microscopic photographs underwent computer-aided image analysis using a computer coupled with an Olympus BX53 optical microscope (Olympus, Tokyo, Japan) equipped with a digital Olympus ColorView IIIu camera (Olympus). The measurements were taken using Cell ${ }^{\wedge} \mathrm{A}$ software (Olympus Soft Imaging Solution GmbH, Münster, Germany).

The expression of PSA, HMWCK, CK-7, CK-20, E-cadherin, COX-2, and mPGES-1 was assessed using a modified semiquantitative IRS scale according to Remmele. This method takes into account both the proportion of the positively stained cells and the intensity of the colour reaction. The score constitutes the product of both parameters and ranges from 0 to 12 points: no reaction -0 points $(-)$; weak reaction $-1-2$ points $(+)$; moderate reaction $-3-4$ points $(++)$; intense reaction $-6-12$ points $(+++)$ [7]. The expression of MCM 7 was evaluated quantitatively by estimating the percentage of positive cells. The endothelial cells of blood vessels were immunostained with von Willebrand factor and examined.

\section{Results and discussion}

Urothelial cancer of prostate gland was diagnosed in both dogs (Fig. 1A, B), and the presence of neoplastic cells in the bladder wall (Fig. 1C) and in the pulmonary veins, which caused pulmonary oedema (Fig. 1D), was also confirmed in one dog.

The neoplastic cells in the prostate gland were oval to spindle-shaped with abundant cytoplasm and 
Table 1. Antibodies used in the study for immunohistochemical stainings. For negative control phosphate-buffered saline (PBS) was used instead of primary antibody; no staining was detected when the primary antibody was omitted

\begin{tabular}{|l|l|c|l|}
\hline Antibody & Type of antibody & Dilution & Positive control \\
\hline CK7 (cytokeratin 7) & Mouse monoclonal anti-human DAKO (Clone OV-TL 12/30) & $1: 100$ & Mammary gland cancer tissue \\
\hline CK20 cytokeratin 20) & Mouse monoclonal anti-human DAKO (clone Ks20.8) & $1: 50$ & Mammary gland cancer tissue \\
\hline $\begin{array}{l}\text { HMWCK(Cytokeratin } \\
\text { High Molecular Weight) }\end{array}$ & Mouse monoclonal anti-human DAKO (clone AE1/AE3) & $1: 50$ & Normal skin tissue \\
\hline $\begin{array}{l}\text { PSA (Prostate-specific } \\
\text { Antigen) }\end{array}$ & Mouse monoclonal anti-human DAKO (clone ER-PR8) & $1: 100$ & Prostate carcinoma tissue \\
\hline E-cadherin & Mouse monoclonal anti-human DAKO (clone NCH-38) & $1: 100$ & Mammary gland cancer tissue \\
\hline Von Willebrand factor & Rabbit polyclonal anti-human DAKO & $1: 100$ & Normal tonsil tissue \\
\hline Ki-67 & Mouse monoclonal anti-human DAKO (clone MIB-1) & $1: 100$ & Mammary gland cancer tissue \\
\hline $\begin{array}{l}\text { MCM7 (Minichromo- } \\
\text { some maintenance } \\
\text { Protein 7) }\end{array}$ & $\begin{array}{l}\text { Mouse monoclonal anti-human Novocastra (clone DCS-141.1), } \\
\text { Novocastra, Newcastle upon Tyne, UK }\end{array}$ & $1: 30$ & Mammary gland cancer tissue \\
\hline $\begin{array}{l}\text { COX-2 (Cyclooxygen- } \\
\text { ase-2) }\end{array}$ & $\begin{array}{l}\text { Rabbit monoclonal anti-human BioSB (clone RBT-COX2), BioSB, } \\
\text { Santa Barbara, CA, USA }\end{array}$ & $1: 50$ & Mammary gland cancer tissue \\
\hline $\begin{array}{l}\text { mPEGS-1 (Microsomal } \\
\text { Prostaglandin E2 Syn- } \\
\text { thase-1) }\end{array}$ & $\begin{array}{l}\text { Rabbit polyclonal anti-human Acris Antibodies (Acris Anti- } \\
\text { bodies, Zelle, Germany) }\end{array}$ & $1: 50$ & Normal tonsil tissue \\
\hline
\end{tabular}

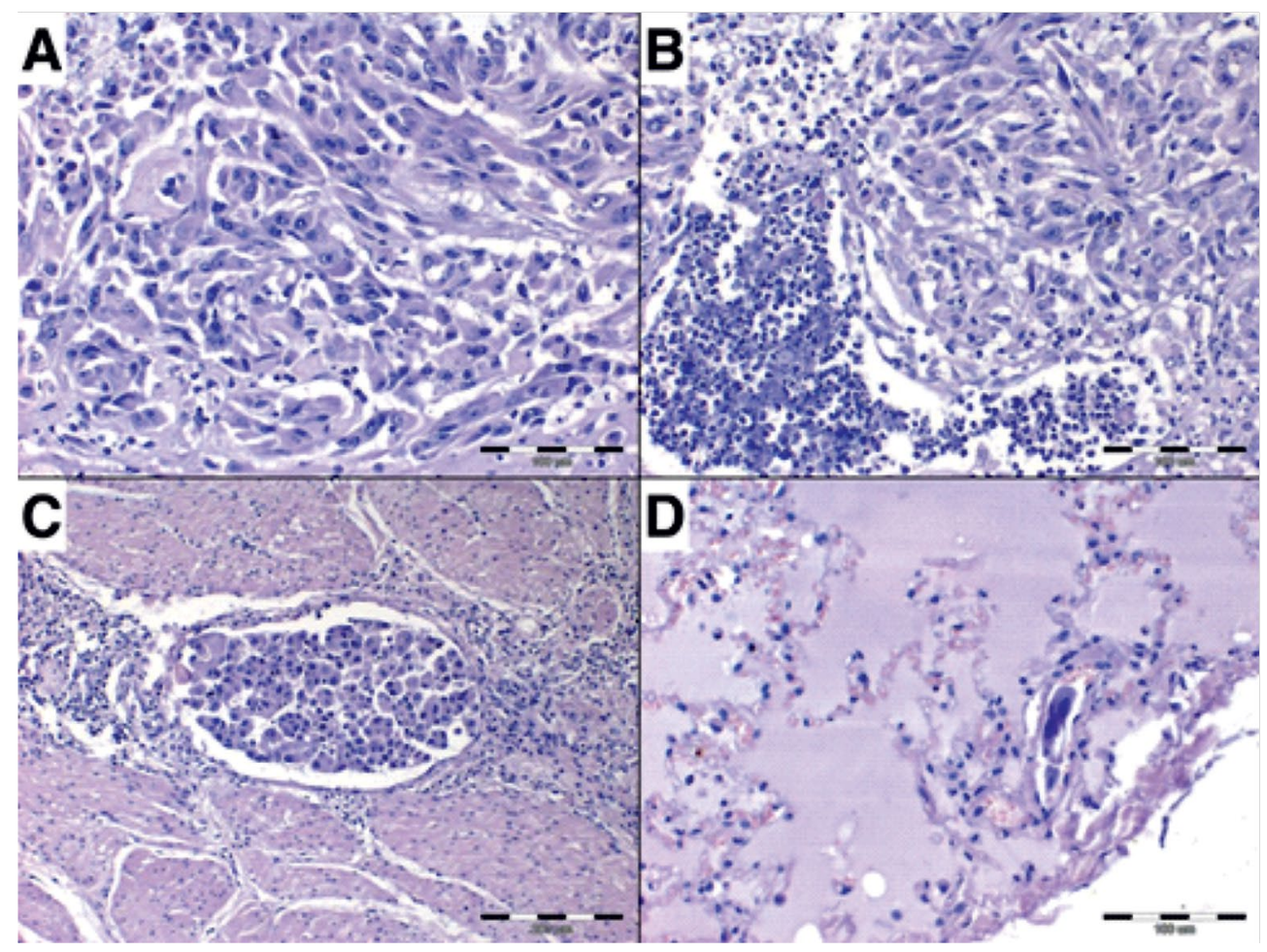

Figure 1. Histological features of the studied canine prostate tumours. A. Urothelial cancer of prostate gland. B. Urothelial cancer of prostate gland with a prominent neutrophil infiltrate. C. A metastatic focus of urothelial cancer of prostate gland in the bladder wall. D. Urothelial cancer of prostate gland cells in the lumen of lung blood vessels. H\&E staining. Magnifications: A, B, D $-200 \times, C-100$. 
visible pleomorphic nuclei with numerous mitotic figures. The cells were arranged chaotically. There were occasional cell clusters surrounded by connective tissue, containing thin-walled blood vessels. Inflammatory cell infiltrates of neutrophils and necrotic foci were also present (Fig. 1B). Small metastatic foci of urothelial cancer of prostate gland were found in the bladder wall, consisting of spindle-shaped and round cells with hyperchromatic nuclei and abundant, clear cytoplasm. These foci were surrounded by a prominent inflammatory infiltrate consisting of lymphocytes and neutrophils. Single urothelial cancer of prostate gland cells were also found in the lumen of lung blood vessels.

The immunohistochemistry results were assessed using the Remmele scale. The strongest expression (8 points) was noticed in the case of heavy cytokeratin (Fig. 2A), while the expression of CK-7 (6 points) and CK-20 (4 points) was slightly weaker (Fig. 2B and $2 \mathrm{C})$. The expression of E-cadherin was evaluated at 8 points in all the studied tumours (Fig. 2D). Von Willebrand factor stained on average eight blood vessels in each field of view. The significant number of vessels in the primary tumour is a sign of tumour potency to form new blood vessels, suggesting higher aggressiveness (local infiltration) and higher metastases formation (Fig. 2E). The expression of COX-2 was graded at 12 points (Fig. 2F), and of mPGES-1 at 8 points (Fig. $2 \mathrm{G}$ ). MCM 7 antigen, used as a marker of cell proliferation, showed an elevated expression manifested by a positive reaction in $80 \%$ of cells (Fig. $2 \mathrm{H}$ ). No PSA expression was observed in the studied sections.

A diagnosis of prostatic cancer in dogs is frequently more complicated than in humans due to the difficulties associated with an intravital prostate biopsy. This is caused by a challenging topographical approach to the prostate, the need for general anaesthesia and challenges of a core-needle biopsy technique. Therefore, prostatic carcinomas in dogs are often diagnosed during a post-mortem examination but may also be found following surgical resection of a part of the prostate or by chance during routine examinations, e.g. during routine vaccination or at the first symptoms of a disease. In veterinary literature, few studies are using new cell markers for detecting human prostate cancer in the diagnosis of canine prostatic carcinoma.

HMWCK and CK7 were found to be the most specific types of cytokeratin for urothelial cancer of prostate gland in dogs [5]. We observed a strong expression of the above-mentioned cytokeratins both in the primary tumour and in the metastatic foci. These are the most specific proteins used in immunochis- tochemical studies during the diagnosis of epithelial origin, routinely used in human medicine during the diagnosis of non-keratinized epithelium, e.g. pseudostratified and ductal epithelium, mesothelium and urothelium. This confirmed that they were cells of the same epithelial origin in the metastatic foci.

The presence of E-cadherin, a protein indirectly responsible for cell adhesion [8], is significant in terms of a tumour metastatic potential. A decrease in the expression of this protein may signify weaker tumour cell connections, allowing the cells to migrate and form metastatic foci. Von Willebrand factor, which is a marker of vascular endothelial cells, was found in a significant number of vessels in the primary tumour, which is closely related to its progression and worse prognosis [9].

Cyclooxygenase-2 (COX-2) and the microsomal prostaglandin E synthase (mPEGS-1) are actively involved in the synthesis of prostaglandins of the $\mathrm{E}$ series (PGE). That, in turn, increases the aggressiveness and invasiveness of the tumour. In normal canine prostatic cells, there was no detectable COX-2 [9] or mPEGS-1 immunoreactivity (Ir), or mPEGS-1-Ir was very low. We found a strong COX-2 immunohistochemical expression in the neoplastic cells.

mPEGS-1 was strongly expressed in the cell cytoplasm of the urothelial cancer of prostate gland. Our results were similar to those of Finetti et al. [10], who studied expression of this protein in humans and found that it produced a strong positive immunohistochemical response. This may indicate that $\mathrm{COX}-2$ and mPEGS-1 play a role in prostate carcinogenesis both in humans and animals.

The urothelial cancers of prostate gland we analysed had a high mitotic index confirmed by $\mathrm{H} \& \mathrm{E}$ staining and strong expression of MCM7. We used MCM7 to assess the proliferative potential and the degree of malignancy of the tumour and its metastases. This protein is part of the minichromosomal maintenance complex that plays a key role in DNA replication during $\mathrm{S}$ phase. Together with the data on the expression of other proteins, we gathered information on the malignancy of this type of tumour and its metastatic potential [9].

No PSA expression was detected. The prostate-specific antigen is a protein that is widely used to confirm the origin of the prostate gland cancer and its metastasis. It is routinely used in human medicine. In our study, neoplastic cells are derived from urothelial epithelium, which is PSA negative. Other authors also found no expression of PSA or only a weak one in canine prostatic carcinoma. Lai et al. reported PSA expression in 8 out of 20 cases of canine prostatic cancer [5]. 


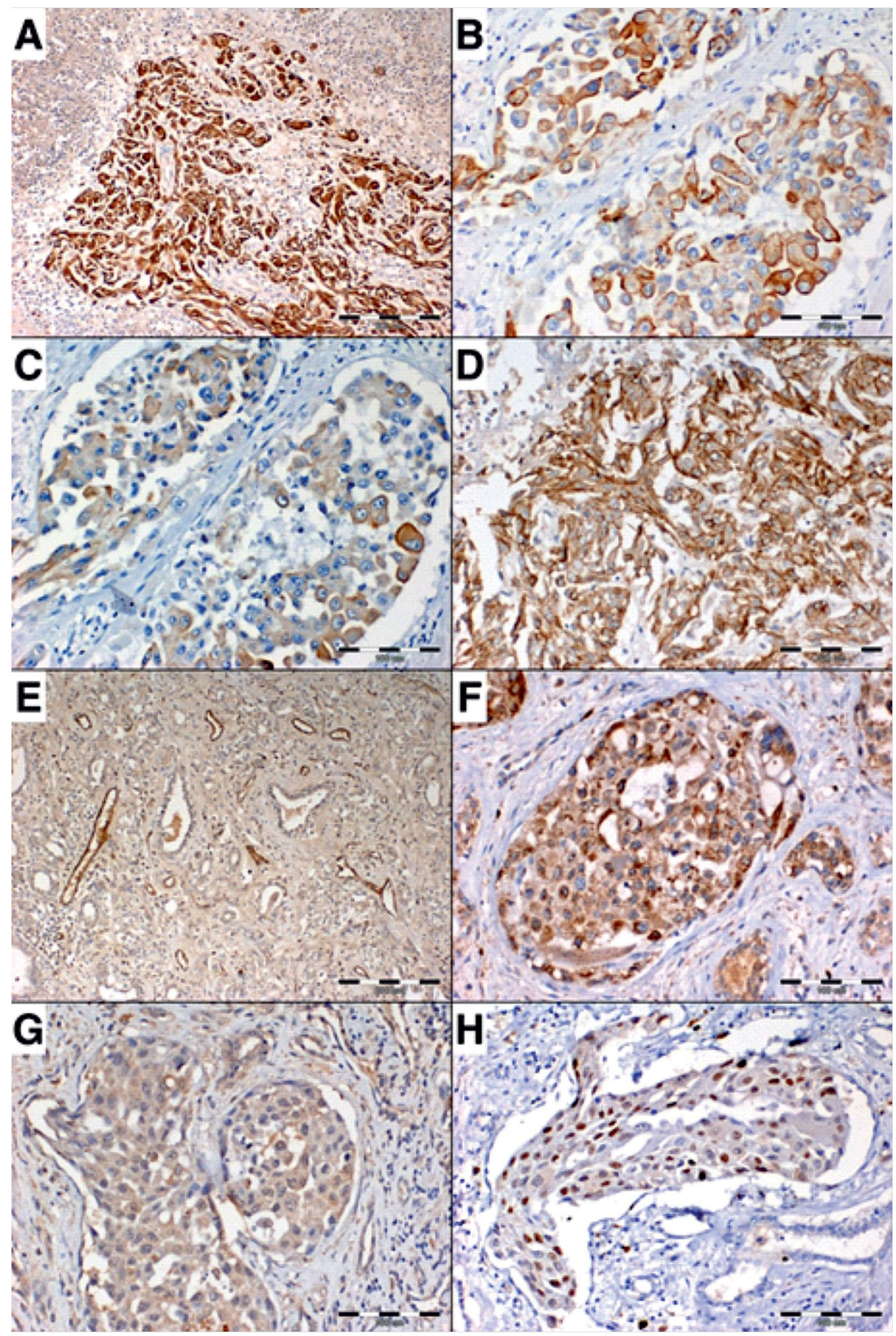

Figure 2. Immunoexpression (brown color) of specific proteins in urothelial cancer of the prostate gland in dog. Cytoplasmic expression of high molecular weight cytokeratins (A), cytokeratin 7 (B) and cytokeratin 20 (C). Plasma membrane expression of E-cadherin (D). Expression of von Willebrand factor in vascular endothelial cells (E). Cytoplasmic expression of cyclooxygenase-2 (F). Cytoplasmic expression of microsomal PGE2 synthase-1 (G). Nuclear expression of minichromosome maintenance complex component 7 (H). Immunohistochemical stainings were performed as described in Methods. 
In this study, we demonstrated the expression of neoplastic markers in a very rare urothelial cancer of prostate gland. The use of immunohistochemistry enabled a much more precise diagnosis of the tumour than the histological examination. It allowed us to determine the tumour type and its degree of malignancy. Our results may contribute to the early diagnosis of urothelial cancer of prostate gland and its early treatment.

\section{References}

1. Yancik R. Cancer burden in the aged: an epidemiologic and demographic overview. Cancer. 1997; 80(7): 1273-1283, doi: 10.1002/(SICI)1097-0142(19971001)80:7<1273:AID-CNCR13 > 3.0.CO;2-4, indexed in Pubmed: 9317180.

2. Waters DJ, Bostwick DG, Waters DJ, et al. Prostatic intraepithelial neoplasia occurs spontaneously in the canine prostate. J Urol. 1997; 157(2): 713-716, doi: 10.1002/(sici)10970045(19970201)30:2<92::aid-pros4>3.0.co;2-m, indexed in Pubmed: 8996404.

3. Waters D, Sakr W, Hayden D, et al. Workgroup 4: Spontaneous prostate carcinoma in dogs and nonhuman primates. The Prostate. 1998; 36(1): 64-67, doi: 10.1002/(sici)10970045(19980615)36:1<64::aid-pros12>3.0.co;2-g.

4. Cornell KK, Bostwick DG, Cooley DM, et al. Clinical and pathologic aspects of spontaneous canine prostate carcino- ma: a retrospective analysis of 76 cases. Prostate. 2000; 45(2): 173-183, doi: 10.1002/1097-0045(20001001)45:2<173::aidpros12>3.0.co;2-r, indexed in Pubmed: 11027417.

5. Lai CL, van den Ham R, van Leenders G, et al. Histopathological and immunohistochemical characterization of canine prostate cancer. Prostate. 2008; 68(5): 477-488, doi: 10.1002/ pros.20720, indexed in Pubmed: 18196537.

6. Kennedy PC, Cullen JM, Edwards JF, et al. Histological classification of tumors of the genital system of domestic animals:tumors of accessory reproductive organs. 2nd edn. World Health Organization Animals. AFIP, Washington, USA; 1998: 53.

7. Brouckaert O, Paridaens R, Floris G, et al. A critical review why assessment of steroid hormone receptors in breast cancer should be quantitative. Ann Oncol. 2013; 24(1): 47-53, doi: 10.1093/annonc/mds238, indexed in Pubmed: 22847811.

8. Ciaputa R. Immunohistochemical and histological features of a spontaneous leydig cell tumour in a rat. Pak Vet J. 2019; 39(04): 603-605, doi: 10.29261/pakvetj/2019.075.

9. Kandefer-Gola M, Nowak M, Ciaputa R, et al. Usefulness of immunohistochemical indicators for diagnosis and prognosis of poorly differentiated tumours. J Vet Res. 2016; 60(3): 323-330, doi: 10.1515/jvetres-2016-0049.

10. Finetti F, Terzuoli E, Giachetti A, et al. mPGES-1 in prostate cancer controls stemness and amplifies epidermal growth factor receptor-driven oncogenicity. Endocr Relat Cancer. 2015; 22(4): 665-678, doi: 10.1530/ERC-15-0277, indexed in Pubmed: 26113609.

Submitted: 26 August, 2020

Accepted after reviews: 2 December, 2020 Available as AoP: 21 December, 2020 